\title{
NTRK Family Gene Mutation
}

National Cancer Institute

\section{Source}

National Cancer Institute. NTRK Family Gene Mutation. NCI Thesaurus. Code C150437.

A change in the nucleotide sequence of an NTRK family gene. 